\title{
Alpháaetifạes Sadiregifter.
}

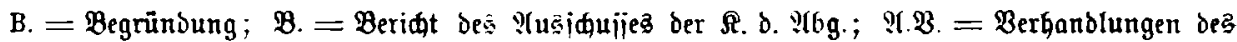

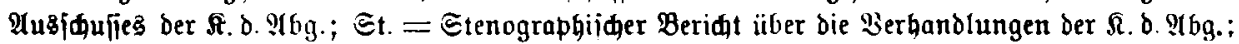

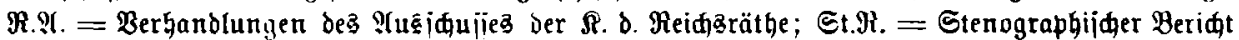

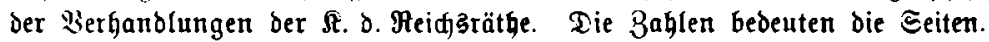

Abgebrannteas Bebäuse, $\mathfrak{B i e b e r a u f b a u , ~} \mathfrak{u n}_{n}=$

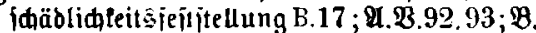
$180-182 ; \mathfrak{A} . \mathfrak{B} .251$; It $219-221,249$,

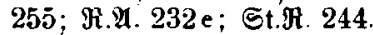

Abjtimmung in ber Sammer ber Mbgeorbneten $226,256,257$ - in ber sammer ber

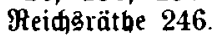

Abtretung zu ijīentlidem 8wede B. 11; $\mathfrak{A} . \mathfrak{B}$. 122 -126; 8.148 ; હt. $196,197$.

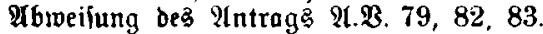

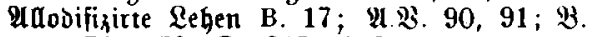
$176-179$; St. $215-218$.

Inrechnurg Der $\mathfrak{B}$ ertberhöhung auf bie $\mathfrak{B e r t h}=$ minderung $\mathrm{B} .11 ; \mathfrak{A} . \mathfrak{B} .66,122-126 ; \mathfrak{B}$. 148; R.R. 232 c.

Intragsinhalt und 2 intragäbelege $\mathfrak{A} \mathfrak{B} .32,52$, $67-72$; $8.159-161$; St. $200-202$.

Alufiorberung ber Beredigten ïber bie $\mathfrak{A} u \underline{B}=$ gleiqung der $\mathfrak{B}$ erthminderung B. 12, 13; Û. B. 78, 79; B. 161, 162, 167, 168-170; St. 204-206.

Aufforderung Der Beredtigten zur Creflätung über ben hinterlegten Betrag B. $15 ; \mathfrak{A}:$ $86,87: \mathfrak{B} .172,173$; . $209-211$.

Aujhebung ber bem jeweiligen (Eigenthümer eineg Brunditïts juftehenden ilecte an

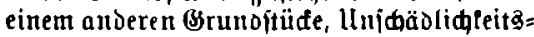
feffitellung B. 17: $\mathfrak{A} . \mathfrak{B} .45,92$; St. 218 .

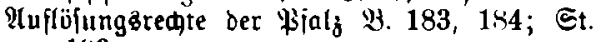
193.

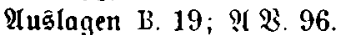

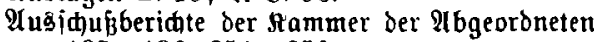
$137-186,250-252$

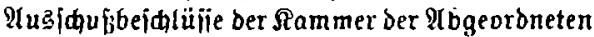
I. Leefung 109-120, II. Reiung 135, 136 .

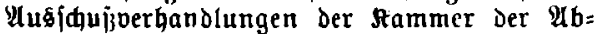
geotoneten, I. Lejung 21-108, Il. Lejung 121-136 - Der fiammer ber \$eidgäräthe $232 \mathrm{a}-232 \mathrm{f}$.

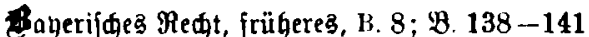
befreiung bes Trennftüd Beilpuntt B. $14 ; 91 . B .14,33,85,86$; ib. 171.

Begrünoung Des E:
Befanntmatung der Auşgleitung an bie Beredtigten B. $13 ; 9 . B .76-78$; B. 166, 167.

Bergredt $\mathfrak{A} . \mathfrak{B} .54 ;$ H. 153.

Bertat Des Mbgeoroneten von $\mathfrak{B a}$ alter 121 **.

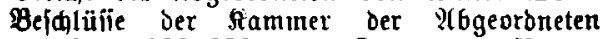

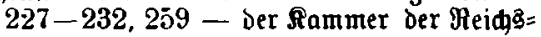
räthe $247,248$.

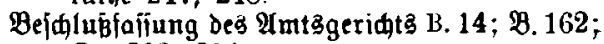
St. $203,204$.

Befaränft perïönliđe Dienftbarteiten B. 10.

Beidwerde der Lajtenbereditigten, ausgeidlojien B. $14 ; \mathcal{M} . \mathfrak{B} .31,36,38,39,41,42,84,85$. B. 170, 171; St. 190, 191, 207; St.FF; 239.

Bemeizmitte1 $\mathfrak{1} . \mathfrak{B} .25,26$

Budertzinfe B. 18; $\mathscr{1} . \mathfrak{3} .95$; 仓t. 224 .

(CÉ)haftreidgnifie $9 . \mathfrak{B} .35,36$.

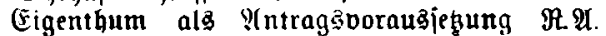
$232 \mathrm{~d}$.

Figenthümerhypothet B. 15; B. 174, 175; St. $223,224$.

(Figenthumol edit, Inhalt B. 137; હt. 187, 188.

Entwari Des (Selepez 2-6.

(Erllärung ber $\mathfrak{L a f t e n b e r e d t i g t e n ~ b e z i i g l i đ a ~} \mathfrak{A} u \mathfrak{s}=$ glethung der Werthminderung B. 12, 13; U. B. $32,33.66,67,130,131$; B. 157, 170.

Erviggeld $\mathscr{M} . \mathfrak{B} .33 ; \mathfrak{Y} .146$; St $\Re .234$.

Jamilien fideifommifie B. 16. 17; $\mathscr{Y} . \mathfrak{B} 35,45$, $90,91,132-134: \mathfrak{B} .150,151,176-179$; St. $215-218$; St.M. 242.

F̃ormelle Behandlung bes Befefentwurfa $\mathfrak{A} . \mathfrak{B}$. $21-24$

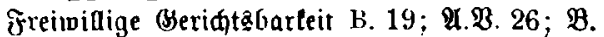
$145,146$.

(15ebühren B. $19 ; 9$. : 35,$96 ; 9.185$; St. 225 .

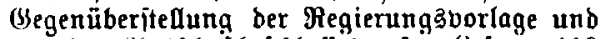

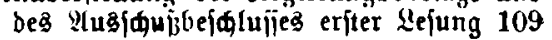
-120 .

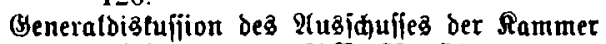
Der Abgeordneten $\mathfrak{A} . \mathfrak{B}$. $25-51$.

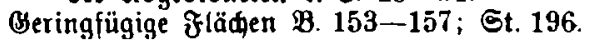




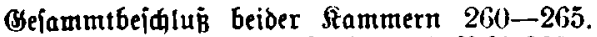
Bejammthafterde (S) runditiide B. $11 ; \Re . \Re .232 \mathrm{c}$. (Selépeastert 266-271.

Bliederuna dez Beriahreng : $:$ ㄱ. 30, 31, 34, $36,42,43,51,55,65,66,73,74$

(Hrundbuhamt, ßrüfung 13. $14 ; \mathfrak{A} . \Re .74 .80$

Brundoiemitbarteiten $\mathrm{B} 10 ; \mathfrak{A} \mathfrak{Y}$. 33. 45, 46, $52,53,54 ; \mathfrak{B} .146,147,152,153 ; \Xi_{t}$.

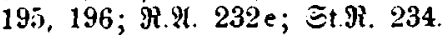

Hipotgeterneuerung $\mathfrak{B} .134$.

Aomplertajten $\mathscr{2} . \mathfrak{B} .30,34$.

Eanowitthị gajtlide Erbqüter B, 16: $\mathfrak{A} .3 .90$. $91,132-134 ; 8.176-179 ;$ ङt. $215-218$.

Laitenberedtiate, Deren Sereinziegung $\mathfrak{A} . \mathfrak{B} .31$, $36,41,50$, i. aud Beínwerde.

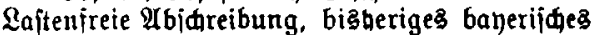
Redt B. 7-10; B. 138-141 - nad bürgerlidem Beießbud B. 6-8- Bor= behalte für bie Sandeägejeggebung B. 8; B. 142.

Lehen B. $16 ; \mathfrak{A} . \mathfrak{B} .90,91 ; \mathfrak{B} .176-179$; St. $215-218$.

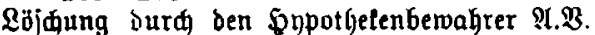
$26-28$.

24utbrewt $2.2 B .54 ; \mathfrak{B} .153$.

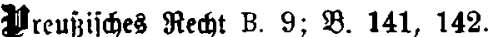

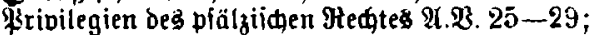
B. 144 , 145; St. 190.

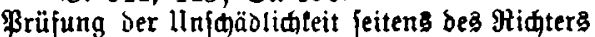
I.:. 42.

Reađaiten B. 18; भ.:2. 29, 30-32, 35, 39, 40 ; B. 146 ; St. 189.

Müdfnahme bes binterleaten Retragå I. B. 63, $64 ;$ B. 157 ; St. 197, 198.

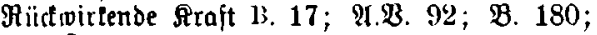
St. 219.

Fadjuerjtändigen. BerneGmuna B.10; $\mathfrak{A} . \mathfrak{B} 42,50$. Eprade des sejepes $\mathfrak{A} . \mathfrak{B} .36,38,41$.

Stenographiider Beridt der Berfandlungen Der Siammer Der $\mathcal{H} b g e o r \delta n e t e n ~ 187-227$, 248, 249, 252 -259 - Der flammer Der Reid)gräthe $233-246$.

Tauith B. 10, 11

Titel dos (Hejetieg $2 . \mathfrak{B} .30,34,36,3 \overline{7}, 38,40$, 50,$96 ; \mathscr{8} .151,186 ;$ ङt. 191

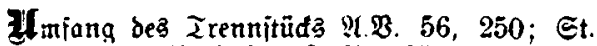
$253 ;: \Re \Re .232$ c; ङt.\%. 235.

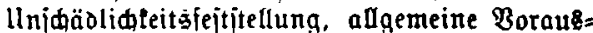
jeģungen $B$. 13: $B$. 152 - gegenūber welden Re edten? B. 10 - im Berbältniffe zu nadber eingetragenen $\Re$ edten $\mathfrak{A} . \mathfrak{B} .80$.

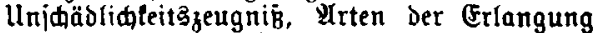
I.B. 43; - Begriji $\mathscr{Q} . \mathfrak{B} .23,81,83$; - Sharafter St. 192 - gleim Röidunagas Bemilligung $\mathfrak{A} . \mathfrak{B}$. $50,80,82,86 ; \mathfrak{B}$. 145 . 172; 91 3. 251, 252: 仓t. 190, 207, 208,

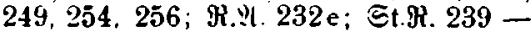
Inbalt $21.3 .33,42,43,57,73,81-$ Beit ber Ertheilung $2 . \mathfrak{B}, 73,80,81$; अ. 162-161; - 3eitpunlt ber $B$ irt = iamleit St, 207-209.

201 U. 3 . 37, 43, 44, 48-50, 64, 87-89; 131,$132 ; \mathfrak{B}$. 148, 149, 173-175; St. 211-213; ङ $\mathfrak{A}$. 241.

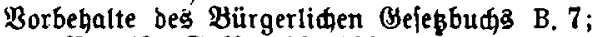
B. 142 ; St. $\Re$. $188,189$.

Borbemerlung 1.

Borfaufôredise B. 15, 16; $\mathfrak{A} .3 .31,32,34,35$, $44,45,51,89 ; \mathfrak{B}, 149,150,175,176$;

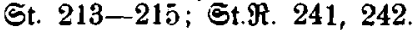

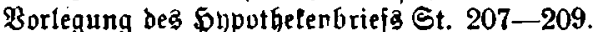

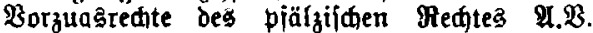
$25--29,94,95 ; \mathfrak{B} .144,145,184$; St. $190,193,224$.

Z11 egveräuberung, Begrifi B. 10.

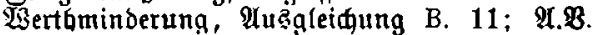
$46-48 ; \mathfrak{B} .154 \mathrm{fi.} \mathrm{-} \mathrm{verhältnî̉mäвig}$ geringe $21.23,39,46,47,126-130 ; \mathfrak{B}$. $154-157,158,251$; $\Re .2 .232 \mathrm{c}$; St. $248,249,254$; Et $\Re$. 235 ; St. 197,198 , 199 - bon nur 50 MRarf B. $11 ; \mathfrak{T} . \mathfrak{B} .55$, $63 ;$ B. 154 fi. - non $10^{\circ}{ }^{\circ}$ beş Brejammt=

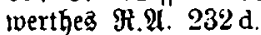

Zujtändiạteit B. 10, 18; 2.P. 94; B. 183; ङt. $223 ; \Re . \Re .232 b$.

3uitellungen $\mathfrak{A} . \mathfrak{B} .131 ; \mathfrak{B} .166,167,171$; St. $203,208-213$.

3wangsenteignung $1 . \mathfrak{B} .29,40,53,93,94$; B. $146,182,183$; . $190,221,222$. 